\title{
SMART ANTENNAS FOR WIRELESS COMMUNICATION SYSTEMS
}

\author{
Anantha Bharathi \\ CVR College of Engineering, Dept of ECE, Ibrahimpatan, R.R.District, A.P., India \\ Email:bharathig8@gmail.com
}

\begin{abstract}
One of the most important challenges with respect to wireless access is the limited capacity of the air interface which is due to the fact that the available transmission bandwidth is finite. Since the number of wireless subscribers is still growing rapidly and the desire for higher data rates is rising - a more efficient use of frequency resources is inevitable to meet future capacity needs. Spatial filtering using adaptive or smart antennas has emerged as a promising technique to improve the performance of cellular mobile systems. This paper presents an overview of smart antennas, including types of smart-antenna systems, and the reasons for their having gained popularity, key characteristics, challenges and benefits, in the context of current and with a view towards future generation multiple input multiple output (MIMO) systems.
\end{abstract}

Index Terms-smart antennas; mobile communication; adaptive arrays; space division multiple access; MIMO.

\section{INTRODUCTION}

Future mobile and wireless applications will require significantly higher data rates and significantly reduced costs per transmitted bit as compared to third generation systems. These requirements on data rate, link quality, spectral efficiency, and mobility cannot be met with conventional single antenna systems. Therefore, antenna arrays and related techniques have been identified as a major area of research.

This paper provides an overview of smart antennas for wireless communications beyond the third generation. Smart antenna systems consist of multiple antenna elements at the transmitting and/or receiving side of the communication link, whose signals are processed adaptively in order to exploit the spatial dimension of the mobile radio channel. Depending on whether the processing is performed at the transmitter, receiver, or both ends of the communication link, the smart antenna technique is defined as multiple input single-output (MISO), single-input multiple-output (SIMO), or multiple-input multiple-output (MIMO). Exploitation of the spatial dimension can increase the capacity of the wireless network by improving link quality through the mitigation of a number of impairments of mobile communications, such as multipath fading and co-channel interference, and by increasing the data rate through the simultaneous transmission of multiple streams by different antennas. Until now, in the design of second- and third generation wireless systems, smart antenna capability was considered as an add-on feature. Adoption of smart antenna techniques in future-generation wireless systems would require the smart antenna feature to be an inherent part of the system design in order to provide the expected beneficial impact on efficient use of the spectrum, enhancement of the quality of service, and realization of reconfigurable, robust, and transparent operation across multi-technology wireless networks.

The paper is organized as follows.

Section 2 presents an insight into smart-antenna systems, using the human auditory system as an analogy. Section 3 discusses challenges in mobile communication system. Section 4 shows how smart antennas can improve the performance of mobile communication systems. Section 5 introduces the different types of smart-antenna systems.

Finally the deployment of Smart antennas in future wireless systems and the implementation issues associated with it are discussed.

\section{SMART ANTENNA ANALOGY}

The functionality of many engineering systems is readily understood when it is related to our humanbody system [1].

Therefore, to give an insight of how a smart-antenna system works, let's imagine two persons carrying on a conversation inside a pitch-dark room (refer to Figure 1). The listener among the two persons is capable of determining the location of the speaker as he or she moves about the room because the voice of the speaker arrives at each acoustic sensor - the ear - at a different time. The human signal processor - the brain computes the direction of the speaker from the time differences or delays received by the two ears. Afterwards, the brain adds the strength of the signals from each ear, so as to focus on the sound of the computed direction. Furthermore, if additional 
speakers join in the conversation, the brain can tune out unwanted interferers, and concentrate on one conversation at a time. Conversely, the listener can respond back to the same direction as the desired speaker by orienting his or her transmitter - his or her mouth -towards the speaker.

Electrical smart-antenna systems work the same way, using many antennas instead of the ears, and a digital signal processor instead of the brain (refer to Figure 1). Therefore, after the digital signal processor receives the time delays from each antenna element, it computes the direction-of-arrival (DOA) of the signal of interest (SOI). It then adjusts the excitations (the amplitudes and phases of the signals) to produce a radiation pattern that focuses on the signal of interest SOI, while tuning out any signal not of interest (SNOI).
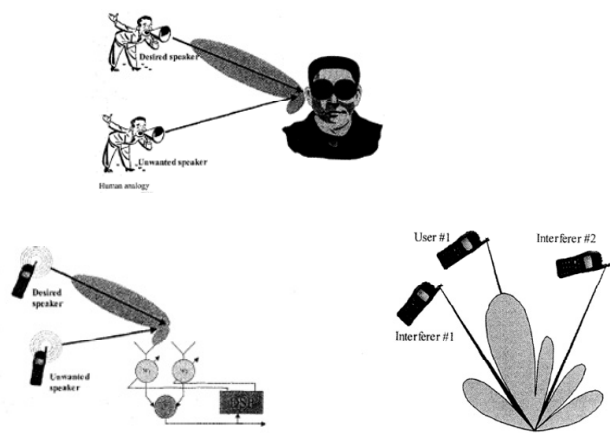

Figure 1. A smart antenna analogy.

\section{PROBLEMS IN MOBILE COMMUNICATIONS}

Multipath propagation, defined as the creation of multiple signal paths between the transmitter and the receiver due to the reflection of the transmitted signal by physical obstacles (Figure 2), is one of the major problems of mobile systems [2].

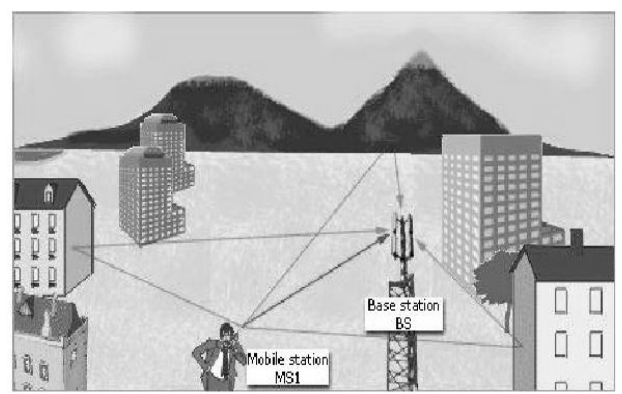

Figure 2. Multipath propagation and co-channel

It is well known that the delay spread and resulting intersymbol interference (ISI) due to multiple signal paths arriving at the receiver at different times have a critical impact on communication link quality. On the other hand, co-channel interference is a major limiting factor on the capacity of wireless systems, resulting from the reuse of the available network resources (e.g., frequency, time) by a number of users.

\section{SMART ANTENNA SYSTEMS}

Many refer to smart-antenna systems as smart antennas, but in reality, antennas are not smart: it is the digital signal processing, along with the antennas, which make the system smart. Although it might seem that smart-antenna systems are a new technology, the fundamental theory of smart antennas is not new. Smart-antenna systems are basically an extension of cell sectoring in which the sector coverage is composed of multiple beams [3]. This is achieved by the use of antenna arrays, and the number of beams in the sector (e.g., a sector of 120") is a function of the array geometry. Because smart antennas can focus their radiation pattern toward the desired users while rejecting all unwanted interference, they can provide a greater coverage area for each base station. Moreover, because smart antennas have a higher rejection of interference - and therefore, a lower bit error rate (BER) - they can provide a substantial capacity improvement. These systems can generally be classified as either switched-beam or adaptive-array systems.

\section{A. switched-beam system}

A switched-beam system is a system that can choose from one of many predefined patterns, in order to enhance the received signal. It is obviously an extension of cell-sectoring, as each sector is subdivided into smaller sectors. As the mobile unit moves throughout the cell, the switched-beam system detects the signal strength, chooses the appropriate predefined beam pattern, and continually switches the beams as necessary. The overall goal of the switched-beam system is to increase gain, according to the location of the user. However, since the beams are fixed, the intended user may not be in the center of the main beam. If there is an interferer near the center of the active beam, it may be enhanced more than the desired user [4].

\section{B. Adaptive-Array Systems}

Adaptive-array systems, provide more degrees of freedom, since they have the ability to adapt the radiation pattern to the RF signal environment in real time.

In other words, they can direct the main beam toward the pilot signal or signal of interest (SOI), while suppressing the antenna pattern in the direction of the interferers or signals not of interest (SNOIs). To put it simply, adaptive-array systems can customize an appropriate radiation pattern for each individual user. This is superior to the performance of a switched-beam system, as is shown pictorially in Figure 3 (and 
discussed in [5]). This figure shows that not only is the switched beam system not able to place the desired signal at the maximum of the main lobe, but it also shows this system's inability to fully reject the interferers. Because of the ability to control the overall radiation pattern in a greater coverage area for each cell site, adaptive- array systems greatly increase capacity. In the presence of a low-level interference, both types of smart antennas provide significant gains over conventional sectored systems. However, when a high-level interference is present, the interference rejection capability of the adaptive systems provides significantly more coverage than either the conventional or switched-beam system [6]. Adaptivearray systems can locate and track signals (users and interferers), and can dynamically adjust the antenna pattern to enhance reception while minimizing interference, using signal processing algorithms.

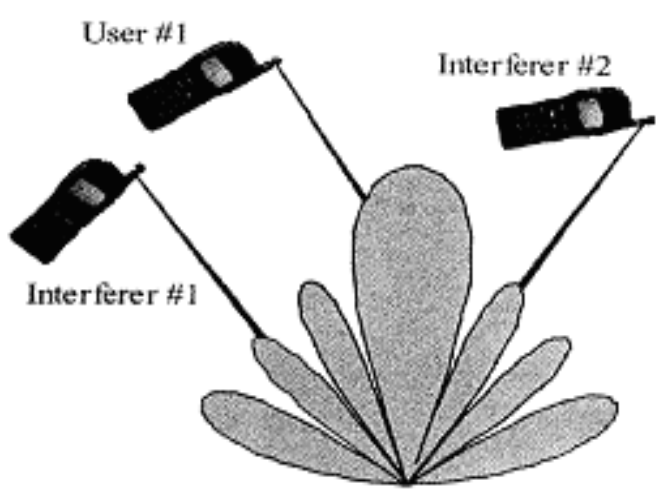

Figure 3(a). switched beam scheme

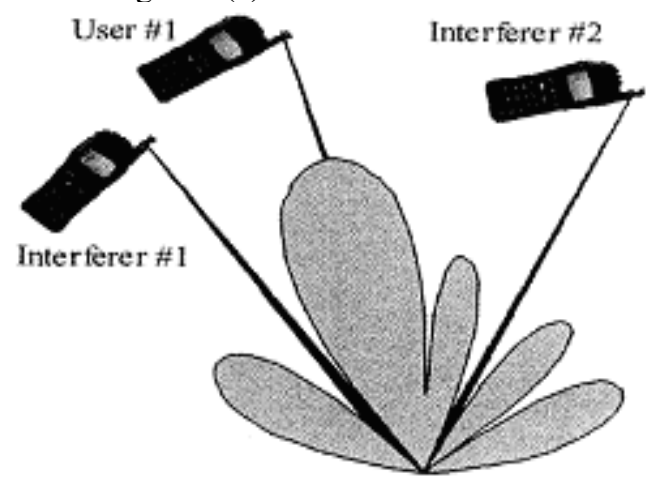

Figure 3(b). adaptive array scheme

A functional block diagram of such a system is shown in Figure 4.

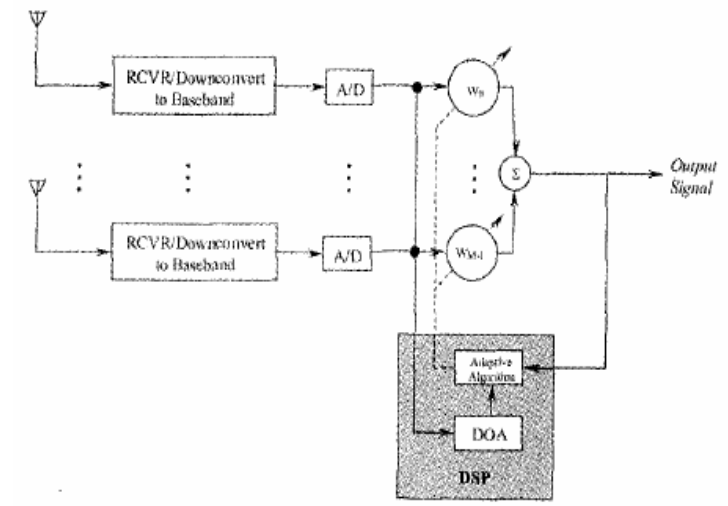

Figure 4. A functional block diagram of such a system

This figure shows that after the system downconverts the received signals to baseband and digitizes them, it locates the signal of interest using the direction-of-arrival (DOA) algorithm. It continuously tracks the signal of interest and signals not of interest by dynamically changing the weights (amplitudes and phases of the signals). Basically, the DOA algorithm computes the direction of arrival of all signals by computing the time delays among the antenna elements. Afterwards, the adaptive algorithm, using a cost function, computes the appropriate weights that result in an optimum radiation pattern. Because adaptive arrays are costly as digital signal processing is involved.

\section{Spatial Division Multiple Access}

Space Division Multiple Access (SDMA) can be used to multiply the capacity given by conventional multiple access techniques such as FDMA, TDMA or CDMA. However, the actual capacity gain which can be achieved with SDMA is highly dependent on the SDMA channel assignment and on the considered scenario (propagation, user distribution, traffic, mobility).

The final phase in the development of cellular radio systems will be SDMA [7,8]. SDMA is among the most sophisticated utilizations of smart-antenna technology: its advanced spatial-processing capability enables it to locate many users, creating a different sector for each user, as shown in Figure 5. This means that more than one user can be allocated simultaneously to the same physical communications channel in the same cell, with only an angular separation. This technology dramatically improves the interference-suppression capability while it greatly increases the frequency reuse, resulting in increased capacity and reduced infrastructure cost. Basically, capacity is increased not only through inter-cell frequency reuse, but also through intra-cell frequency reuse. 


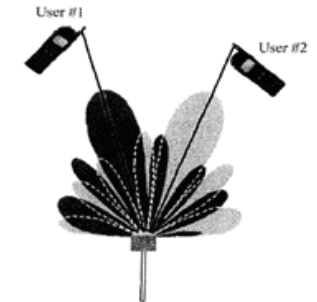

Figure 5. An adaptive array system with SDMA

\section{.D. Smart Antennas Benefits}

The principle reason for the growing interest in smart antenna systems is the capacity increase. In densely populated areas, mobile systems are normally interference-limited, meaning that the interference from other users is the main source of noise in the system. This means that the signal-to-interference ratio (SIR) is much larger than the signal-to-noise ratio (SNR). In general, smart antennas will increase the SIR by simultaneously increasing the useful received signal level and lowering the interference level.

Smart antennas at base stations can be used to enhance mobile communication systems in several ways:

- $\quad$ increased BS range

- less interference within the cell

- less interference in neighboring cells

- $\quad$ increased capacity by means of SDMA

\section{CONCLUSIONS}

This paper gives a general overview of smart antennas. Problems in wireless communications and how they are overcomed using smart antennas is discussed. Even higher spectral efficiencies can be achieved if antenna arrays are not only used at the base station but also at the mobile to create multiple-inputmultiple-output systems. The achievable spectral efficiency, however, depends on the propagation and interference environment. If a rich scattering environment is available, an enormous spectral efficiency can be obtained via spatial multiplexing.

\section{REFERENCES}

[1] International Engineering Consortium, Smart Antenna Systems, an on-line tutorial, http://www.iec.orglonline/tutorialslsma ant/index.html.

[2] A.Paulraj, R.Nabar, and D.Gore, Introduction to SpaceTime Wireless Communications, Cambridge Univ. Press, 2003.

[3] B. Pattan, Robust Modulation Methods \& Smart Antennas in Wireless Communications, Upper Saddle River, NJ, Prentice Hall PTR, 2000
[4] M. Chryssomallis, "Smart Antennas," IEEE Antennas and Propagation Magazine, 42, 3, June 2000, pp. 129136

[5] Choi Seungwon, Shim Donghee, and T. K. Sarkar, "A Comparison of Tracking-Beam Arrays and SwitchingBeam Arrays

[6] Operating in a CDMA Mobile Communication Channel," IEEE Antennas and Propagation Magazine, 41, December 1999, pp. 10-56.

[7] G. L. Stuber, Principles of Mobile Communications, The Netherlands, Kluwer Academic Publishers, 1996.

[8] G. V. Tsoulos, "Experimental and Theoretical Capacity Analysis of Space-Division Multiple Access (SDMA) with Adaptive Antennas," IEE Proceedings on Communications, 146, 5, October 1999, pp. 307-31. 